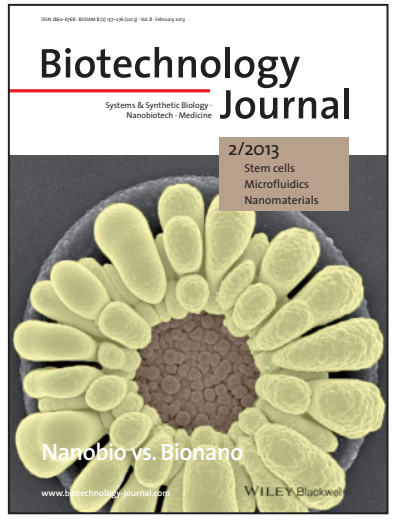

Cover illustration

Special issue:

Nanobio vs. bionano

As researchers develop capabilities to engineer and manipulate objects and systems at ever-diminishing scales, we are now entering the realm of scientific discoveries at the nano-level. One such invention is pictured on the cover of this Special Issue, edited by François Baneyx (University of Washington, USA) and Je-Kyun Park (KAIST, Korea), showing a single-cell-sized neural electrode with unique gold nanograin structures in the beautiful shape of a sunflower. Image: (C) Yoonkey Nam

Biotechnology Journal - list of articles published in the February 2013 issue.

Editorial

Nanobio versus Bionano - what's in a name?

François Baneyx and Je-Kyun Park

http://dx.doi.org/10.1002/biot.201200407

Commentary

Functional nanoscale biomolecular materials

Jonathan S. Dordick

http://dx.doi.org/10.1002/biot.201200338

\section{Review}

Arrayed cellular environments for stem cells and regenerative medicine

Drew M. Titmarsh, Huaying Chen, Ernst J. Wolvetang and Justin J. Cooper-White

http://dx.doi.org/10.1002/biot.201200149

Review

Microfluidic systems: A new toolbox for pluripotent stem cells Sasha Cai Lesher-Perez, John P. Frampton and Shuichi Takayama

http://dx.doi.org/10.1002/biot.201200206

Review

Microfluidic tools for developmental studies of small model organisms - nematodes, fruit flies, and zebrafish Hyundoo Hwang and Hang Lu

http://dx.doi.org/10.1002/biot.201200129

Research Article

Gold nanograin microelectrodes for neuroelectronic interfaces Raeyoung Kim, Nari Hong and Yoonkey Nam

http://dx.doi.org/10.1002/biot.201200219

Research Article

Self-assembled DNA-based giant thrombin nanoparticles for controlled release

Jong Hwan Sung, Daehoon Han and Jong Bum Lee

http://dx.doi.org/10.1002/biot.201200312
Research Article

An autonomously self-assembling dendritic DNA nanostructure for target DNA detection

Harish Chandran, Abhijit Rangnekar, Geetha Shetty, Erik A. Schultes, John H. Reif and Thomas H. LaBean http://dx.doi.org/10.1002/biot.201100499

Research Article

Engineering protein filaments with enhanced thermostability for nanomaterials

Dominic J. Glover, Lars Giger, Jihyun R. Kim and Douglas S. Clark

http://dx.doi.org/10.1002/biot.201200009

Research Article

Biologically inspired strategy for programmed assembly of viral building blocks with controlled dimensions Jennifer M. Rego, Jae-Hun Lee, David H. Lee and Hyunmin $Y i$

http://dx.doi.org/10.1002/biot.201100504

Research Article

Bionanotechnology application of polypeptides in a hair color product: Self-assembly enables expression, processing, and functionality

Pierre E. Rouvière, Jing Li, Donald J. Brill, Lisa D. Reiss, Timothy R. Schwartz, Lisa A. Butterick, Stephen R.

Fahnestock and Tanja M. Gruber

http://dx.doi.org/10.1002/biot.201200008

Rapid Communication

Size-modulated synergy of cellulase clustering for enhanced cellulose hydrolysis

Shen-Long Tsai, Miso Park and Wilfred Chen

http://dx.doi.org/10.1002/biot.201100503

Research Article

Addressable self-immobilization of lactate dehydrogenase across multiple length scales

Sibel Cetinel, H. Burak Caliskan, Deniz T. Yucesoy,

A. Senem Donatan, Esra Yuca, Mustafa Urgen,

Nevin G. Karaguler and Candan Tamerler

http://dx.doi.org/10.1002/biot.201100502 
Review

\title{
Microfluidic systems: A new toolbox for pluripotent stem cells
}

\author{
Sasha Cai Lesher-Perez ${ }^{1}$, John P. Frampton ${ }^{1}$ and Shuichi Takayama 1, 2, 3 \\ ${ }^{1}$ Department of Biomedical Engineering, University of Michigan, Ann Arbor, MI, USA \\ 2 Department of Macromolecular Science and Engineering, University of Michigan, Ann Arbor, MI, USA \\ ${ }^{3}$ Division of Nano-Bio and Chemical Engineering WCU Project, UNIST, Ulsan, Republic of Korea
}

Conventional culture systems are often limited in their ability to regulate the growth and differentiation of pluripotent stem cells. Microfluidic systems can overcome some of these limitations by providing defined growth conditions with user-controlled spatiotemporal cues. Microfluidic systems allow researchers to modulate pluripotent stem cell renewal and differentiation through biochemical and mechanical stimulation, as well as through microscale patterning and organization of cells and extracellular materials. Essentially, microfluidic tools are reducing the gap between in vitro cell culture environments and the complex and dynamic features of the in vivo stem cell niche. These microfluidic culture systems can also be integrated with microanalytical tools to assess the health and molecular status of pluripotent stem cells. The ability to control biochemical and mechanical input to cells, as well as rapidly and efficiently analyze the biological output from cells, will further our understanding of stem cells and help translate them into clinical use. This review provides a comprehensive insignt into the implications of microfluidics on pluripotent stem cell research.

Received 16 MAY 2012

Revised 23 AUG 2012

Accepted 25 SEP 2012

Keywords: Bioengineering · Embryonic stem cells · Microfluidics · Pluripotent stem cells

\section{Introduction}

\subsection{Stem cells then and now}

The first pluripotent stem cells were isolated in 1981 from mouse embryos, and were termed embryonic stem cells (ESCs) to distinguish them from pluripotent cells obtained from teratocarcinomas [1]. Since this discovery, advances in our understanding of stem cells have led to a variety of proposed cell-based strategies for improving regenerative medicine, treating degenerative diseases, and discovering novel drug targets. These strategies take advantage of the two defining properties of pluripotent stem cells, their abilities to self-renew indefinitely and to differentiate into

Correspondence: Prof. Shuichi Takayama, Department of Biomedical Engineering, University of Michigan, Ann Arbor, MI 48109, USA E-mail: takayama@umich.edu

Abbreviations: 2D, two-dimensional; 3D, three-dimensional; ATPS, aqueous two-phase system; ECM, extracellular matrix; EB, embryoid body; GMP, good manufacturing practice; (h/m) ESC, (human/mouse) embryonic stem cell; iPS, induced pluripotent stem specific cells from any one of the three germ lines upon appropriate stimulation [1-3].

Early work characterized the cues that regulated stem cell pluripotency and differentiation. ESCs required a variety of factors to maintain their pluripotent status, as they could only be expanded in vitro through the use of feeder cells and conditioned medium [1, 4]. Further characterization revealed that the key to maintaining potency in mouse ESCs (mESCs) was the presence of specific growth factors, whereas differentiation to each germ layer and respective downstream lineages required entirely different sets of growth factors and external stimuli [3].

In the process of characterizing mESCs, an efficient set of tools for genetically engineering mouse models, creating feeder-cell culture systems, and directing cell differentiation were established [5]. However, upon isolation of the first human ESCs (hESCs), an opportunity was opened to shift from basic research on pluripotent stem cells to clinical research. As with mESCs, hESCs displayed dif-

Color online: See the article in color to view the figures in color. 
ferential responses that were dependent on biochemical factors [6-8] as well as a variety of other stimuli. These and other findings triggered a growth in the development of devices for isolating, stimulating, collecting, and analyzing stem cells.

\subsection{What does a device have to offer?}

Traditional methods for culturing stem cells, such as twodimensional (2D) cultures on extracellular matrix (ECM)coated cultureware or on feeder-cell layers, or as three-dimensional (3D) embryoid bodies (EBs), are limited in their ability to apply precise stimuli to individual cells or cell populations. They are also difficult to adapt for highthroughput biochemical or molecular analysis on subpopulations of cells [9].

These limitations can be resolved by using microfluidics to control the cellular microenvironment [10-12]. Microfluidic systems are versatile and provide high spatiotemporal resolution for patterning molecules and cells (for reviews see [10-12]). Since ESCs and other types of pluripotent stem cells display high sensitivity to external stimuli, the high degree of control provided by microfluidics is useful for investigating their biology [13]. In the past few years, several reviews have highlighted the properties, advantages, and future growth areas of microfluidic technologies in stem cell research $[14,15]$.

Our review does not focus on the fundamentals of these fields, but rather encompasses more detailed and specific work applied to pluripotent stem cells, in particular ESCs, cultured in microfluidic environments. In our discussion, we touch upon microfluidic tool sets that have not yet been applied to pluripotent stem cells, but have the potential to provide useful control or analysis methods for use with these cellular populations. This review is organized into several practical areas that microfluidics and other microtechnology platforms are uniquely capable of addressing: (i) Controlled EB formation and co-culture geometry (Section 2); (ii) precise delivery of biochemical and mechanical stimulation to stem cells to direct their differentiation (Section 3); and (iii) acquisition of biochemical data from single cells or small populations of cells (Section 4) (Fig. 1). Although much of the discussion is focused on ESCs, we also describe applications involving other pluripotent and multipotent stem cell types.

\section{EBs and co-cultures}

A variety of culture systems exist for maintaining stem cells in their pluripotent state prior to differentiation. These include 2D substrates where growing ESC colonies are maintained with irradiated feeder cells [2], and 2D cultures without feeder cells that use select ECM proteins or polymer coatings and medium formulations to maintain pluripotency [16, 17]. Maintaining pluripotency in conventional macroscale methods is fairly standardized, although robustly directing differentiation of pluripotent stem cells has been more difficult. Traditional methods for ESC differentiation include formation of 3D EBs [18, 19]. In EBs, ESCs differentiate spontaneously; however, differentiation can be directed down desired lineages by exposure to appropriate developmental cues. These traditional formats, however, suffer from limitations such as the inability to tightly control $\mathrm{EB}$ size and finely regulate spatiotemporal biomolecular cues. Can transitioning to microfluidic culture platforms circumvent some of these limitations? In this section we describe ESC differentiation platforms with a focus on generating EB cultures and generating patterned ESC Co-cultures.

\section{D/3D Devices for Stem Cell \\ Maintenance, Progation, Differentiation and Sorting}
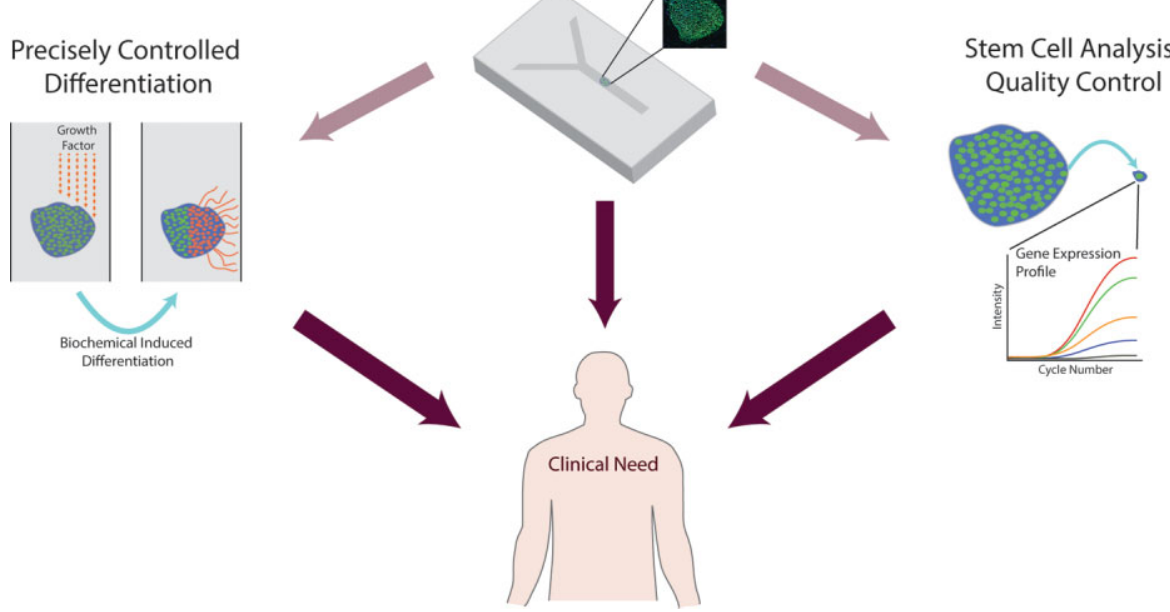

Figure 1. Microfluidics can be used to culture and analyze stem cells. Devices provide precise stimulation to cells and enable analysis of cell properties down to the level of single cells. These systems will aid in clinical translation of cellbased therapies. 


\section{A. Microfabricated Hanging Drop Systems for EB Culture}

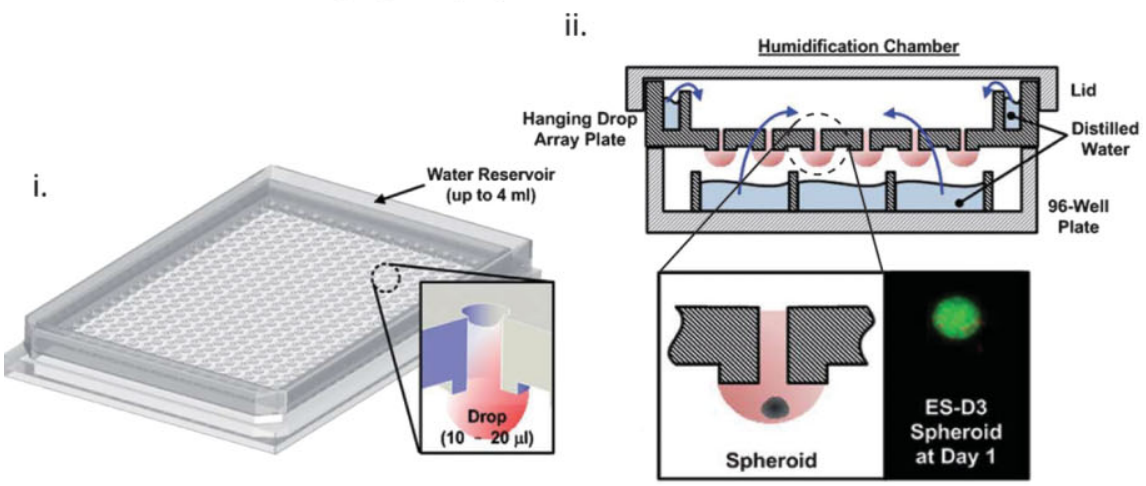

B. Trapping and Docking Devices for EB Formation

i.

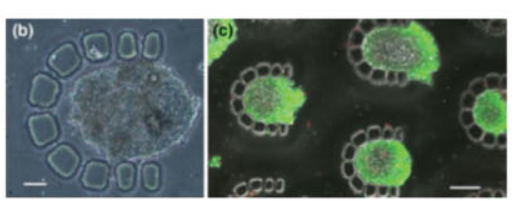

ii.

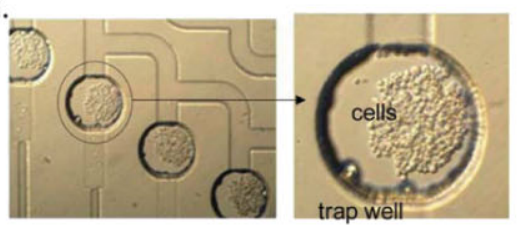

iii.

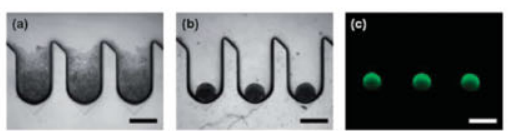

iv.

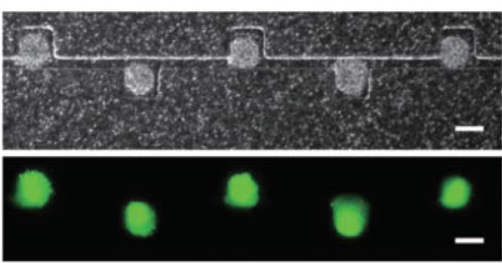

C. Non-Contact Device-Free Patterning of ES Cells

i.

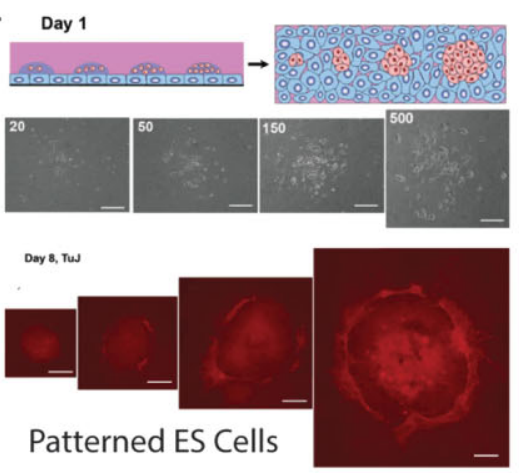

ii.

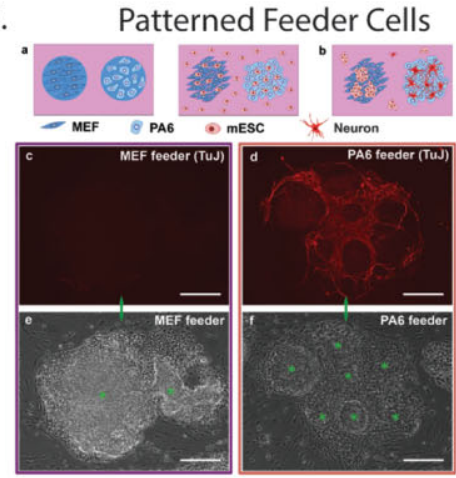

Figure 2. The microfluidic tool set for organizing pluripotent cells as EBs and as co-cultures. (A) Microfabricated plate with specially designed through-holes for high-throughput hanging-drop formation with topside cell and media delivery. The hanging-drop plate allows multiple medium exchanges, reducing problems associated with osmolality and nutrient depletion (reprinted with permission from RSC Publishing [21]). (B) Platforms with various geometries allow trapping of ESCs for formation of EBs. ((i) Reprinted with permission from Springer [28]. (ii) Reprinted with permission from RSC Publishing [24]. (iii) Reprinted with permission from RSC Publishing [26] (iv) Reprinted with permission from RSC Publishing [25]). (C) ATPSs allow ESCs (i, Reprinted with permission from Wiley [34]) and feeder cells (ii) Reprinted with permission from Wiley [35]) to be micropatterned on a variety of substrates to promote differentiation.

\subsection{Microfluidic hanging drop EB culture}

Spheroid cultures were first implemented with pluripotent embryonic carcinoma cells that aggregated in suspension and could subsequently differentiate in vivo into teratomas [18]. These spheroidal cultures were later adapted for use with ESCs (for review see [19]). One of the most commonly used methods for growing EBs is hanging-drop culture. Hanging-drop culture utilizes microscale volumes of culture media, and employs the surface tension of fluid on the interior of Petri dish lids or underside of inverted multi-well plates to contain EBs.
These droplets require small volumes ( $50 \mu \mathrm{L}$ or less) to maintain their stability, but due to the inverted droplet culture format, changing their media and manipulating the system is cumbersome and time consuming. These factors make traditional hanging-drop culture an unattractive method for applications that require many EBs. The strength of this system, however, is its ability to control the number of cells in each EB, minimizing variability in size and quality. By employing microfabrication, researches can capitalize on this strength while minimizing the difficulties associated with the technique itself. 
Normal culture time before nutrient depletion is $\sim 2$ days for hanging drops. Lee et al. [20] developed a method to perform long-term hanging-drop culture of EBs without disturbing autocrine/paracrine signaling through media exchanges. This device improved the ability to supplement nutrients in hanging drops by providing a fluid reservoir, allowing for longer culturing due to the presence of more media. However, this system did not completely eliminate another limiting factor in hangingdrop cultures of ESCs, i.e. the tendency for osmolality changes to occur over time. More recently, a highthroughput hanging-drop array plate was developed, providing access to cultured hanging drops for media exchanges and maintenance of osmolality. This system also provided the ability to supplement molecules, such as growth factors and drugs [21] (Fig. 2A). The plate was fabricated in a 384-well format to interface with liquid-handling robots, allowing high-throughput hanging-drop production and testing.

\subsection{Culturing EBs in microchannels}

Fluid-focusing channels, as well as topographical features and recesses, can also be used to collect and aggregate precise cell numbers in defined regions. Microfluidic entrapment devices can provide continuous perfusion and controlled biochemical or mechanical stimulation (Fig. 2B). Size regulation is critical as EBs have been shown to differentiate down specific lineages as a function of size $[22,23]$. Thus, an immediate benefit of using hanging drops and microchannel devices is tightly regulating EB size [23-26]. These formats result in less variation in biomarker expression as compared to conventional EB suspension cultures where EB size is not controlled [27]. Hwang et al. [23] used micro-wells to create uniformly shaped mESC EBs of specific sizes. Larger EBs showed enhanced cardiogenesis, while smaller EBs had increased endothelial cell differentiation. Beyond demonstrating a difference in morphogenesis, they elucidated WNT signaling involvement in the EB size-dependent differentiation process. Size-selective differentiation was influenced by the noncanonical WNT pathway, where WNT5a and WNT11 regulated the size-mediated differentiation responses towards endothelial and cardiac tissue, respectively. It is likely that parameters such as changes in diffusion of soluble molecules, ECM-cell, and cell-cell interactions also play a role in size-dependent differentiation [22].

Other methods for focused cell trapping have been used for high-throughput investigation of the effects of different media types and specific biomolecules on differentiation [24, 28]. Kim et al. [24] recently demonstrated a microfluidic resistance network in which the number of cells seeded in microchambers could be varied based on duration of cell seeding. This study demonstrated that EBs derived from pluripotent embryonic carcinoma cells could be maintained in a high-throughput format and could be differentiated with medium containing retinoic acid. The retinoic acid treatment promoted expression of neuron-specific biomarkers and neuronal morphology with mature neurites, as compared to EBs exposed to retinoic acid-free differentiation medium that contained only a few cells expressing neural biomarkers. Other systems, such as cell docking platforms, have used microfluidics to automate the EB culture process, allowing EBs to be sequentially formed, differentiated, and plated for postdifferentiation analysis in one device [29].

\subsection{Microfluidic co-cultures}

Since pluripotent stem cells are more easily maintained and differentiated in the presence of support cells, another active area of research is the production of platforms that can control the orientation of co-cultured cells and pattern cells in defined geometries. 3D microfluidic co-culture patterning has been achieved using cell trapping. The system described by Torisawa et al. [30] was used to compare a heterogeneous mixture of two cell types with cells patterned as separate spheroid bodies. Initial analysis of this platform was characterized by observing uniformity of OCT4, a pluripotent marker. Uniform OCT4 loss occurred when the co-cultures were mixed together to form heterogeneous spheroids, whereas the patterned co-culture resulted in asymmetric expression levels of OCT4 in mESC EBs, with regional downregulation of OCT4 adjacent to co-cultured HepG2 cells.

Additionally, biopatterning and bioprinting can be applied to co-culturing, allowing researchers to position cells in patterns on cell culture substrates. Cell bioprinting is accomplished in one of two ways, either by directly depositing the cells in specific regions, or by patterning biomolecules that display preferential cellular adhesion. These methods include dip pen lithography, soft lithography, photolithography, laser-based transferring or laser writing, electroprinting, (micro) extrusion, inkjet deposition, and droplet ejection [31]. Implementation of these techniques to manipulate ESCs has already begun with methods such as droplet ejection used to print highthroughput hanging drops containing mESCs on petri dish lids [32] and photopolymerization to develop cellcompatible polymer microarrays to test hESC growth and differentiation [33]. Another option for biopatterning Co-cultures of stem cells is the use of aqueous two-phase systems (ATPSs) (Fig. 2C). ATPSs can directly pattern cells using only solutions of non-cytotoxic dextran and polyethylene glycol dispensed with either a micropipette or pin tool. It was found that $\mathrm{mESCS}$ printed on the appropriate feeder layer could differentiate into neurons [34]. It was also found that this neuronal differentiation scaled nonlinearly with colony size, as larger colonies produce a greater than expected number of neurons. Additionally, feeder layers could be deposited at specific sites causing 
local differentiation of co-cultured ESCs [35]. This simple and adaptable ATPS technique offers a method for printing and differentiating cells that does not involve a device or specialized culture conditions, making it an attractive tool for stem cell biologists.

\section{Microtechnologies provide instructive stimuli}

Stem cells respond to a variety of external stimuli including fluid forces, biochemical factors, and physical interaction with ECM. In this section we describe how microfluidics can control these stimuli and engineer better in vitro stem cell niches.

\subsection{Directing pluripotent cellular development taking cues from embryogenesis}

Embryos are exposed to fluid flow as they travel through fallopian tubes [36], and to hemodynamic forces during yolk sac vascular remodeling [37], and produce fluid movement by way of their nodal cilia [38]. Although not completely understood, it is likely that mechanosensingbased cues deriving from fluid flow [39] drive the process of embryogenesis in coordination with mixtures of biochemical factors that form gradients in the fluidic environment [40]. Biochemical factors play a pivotal role in organ and tissue development, and act through dosedependent spatiotemporal regulation $[41,42]$. In the developing embryo, gradients of these factors result in welldefined functional zones within tissues [43]. Biochemical factors also regulate the maintenance of pluri/multipotency of stem cells originating from the inner cell mass [44, 45] and control lineage specification [46-48]. Unless specific signals are regulated in terms of dose and spatiotemporal localization, gross morphologic and developmental abnormalities will occur, potentially resulting in embryonic lethality $[49,50]$.

It is well accepted that multiple biochemical mechanisms are involved in the formation of organized tissues [51]. However, it has more recently been appreciated that these biochemical cues are coupled to biomechanical signaling mechanisms. Together, the tensile forces generated when cells adhere to each other, as well as to the ECM, provide a mechanical framework that is transduced into biochemical signals within the cells, resulting in differential responses in cell proliferation, differentiation, and migration [52, 53].

This brief synopsis can provide only a small peek into the multitude of external cues that influence differentiation, pluripotency-maintenance, proliferation, and organization and migration of ESCs. When working with these and other pluripotent stem cells, re-creation of embryogenesis cues are used in an attempt to better study and manipulate these cells [54]. Conventional culturing systems, such as polystyrene tissue culture plates, have been used extensively; however, these systems suffer from an inability to spatially control cell seeding, define cell or protein patterning, temporally control the cell culture environment, and regulate solid and fluid mechanical stimulations. In essence, they fall short of recapitulating tightly defined embryogenesis cues. Microengineered culture systems can be used in a variety of ways to lessen the gap between cell culture and in vivo developmental niches.

\subsection{Utilizing fluid dynamics and fluid forces}

Microfluidics takes advantage of fluid forces on the microscale that can be classified as hydrodynamic, gravitational, capillary, wetting, and adhesion forces. This section primarily discusses the phenomena produced by hydrodynamic forces within microfluidic devices (for a complete review of the other forces see [55]). Due to the length scales of microfluidic channels, the fluid regimes are almost always defined by low Reynolds' numbers, resulting in laminar flow. A benefit of laminar flow is that two or more streams flowing in parallel will not mix, except by diffusion. This property can be used to hydrodynamically pattern materials such as proteins or cells [56]. Laminar flow can also be used to create fluid packets or segmented flow, placing non-continuous fluid types within one another [57]. These fluid packets can provide temporal stimulation to cells. Due to the typically non-turbulent flow in microfluidic devices, particle positioning can be determined via theoretical analysis, defining the flow properties within devices [58] (Fig. 3A).

Using well-defined geometries, shear stress applied to a stem cell culture growing within a device can be precisely controlled. Shear stress applied to ESCs can result in hematopoiesis [59] or differentiation to vascular endothelial cells [60]. Similar findings have been reported using logarithmic microfluidic arrays, where self-renewal and expression of pluripotent markers in ESCs could be maintained using different flow regimes, essentially priming these cells for differentiation by shear stress [61]. Furthermore, providing non-continuous shear stresses to ESCs was shown to induce differentiation into vascular wall cells [62] and vascular grafts [63].

\subsection{Precise biochemical stimulation}

Microfluidics can generate well-defined step-like or smooth gradual gradients for in vitro culture systems [64]. Hydrodynamic compartmentalization can be created above stem cells cultured in simple Y-type channels, where laminar streams form and allow the partitioning of chemical stimuli in distinct regions of the channel (steplike gradient). This phenomenon was used to demonstrate biochemical treatment of a portion of a single hESC colony [65] (Fig. 3B). This study demonstrated that adher- 
A. Controlled Perfusion and Shear

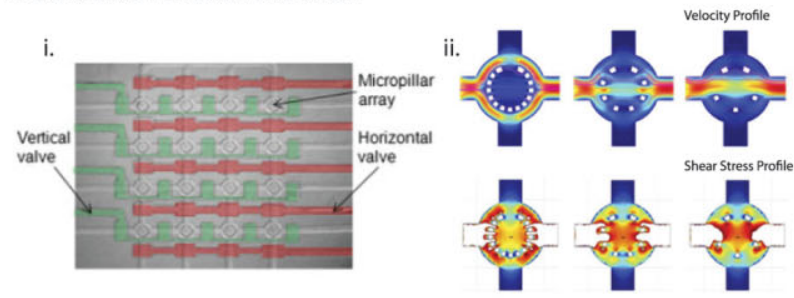

B. Compartmental Biochemical Delivery
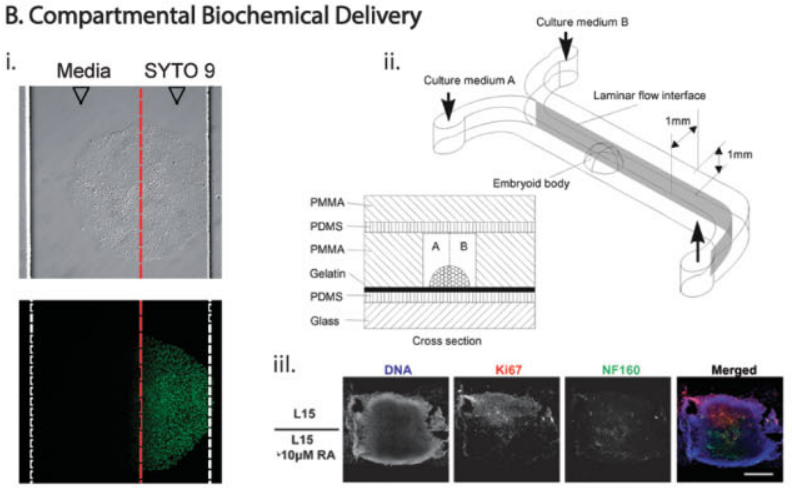

C. Controlled Biochemical Gradients

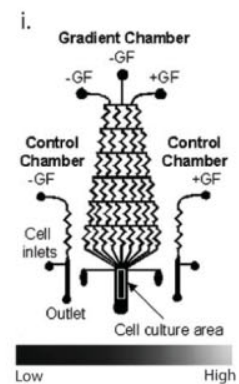

ii.

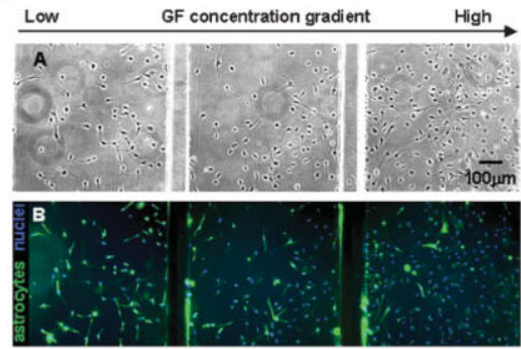

D. Topographic Cues

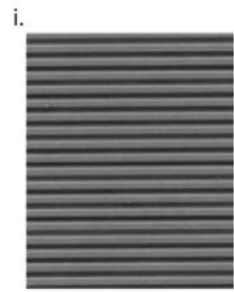

$500 \mathrm{~nm}$ pattern
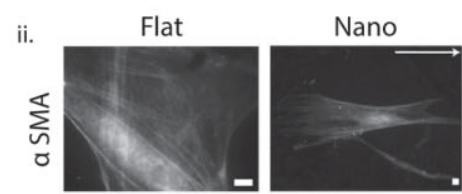

iil.

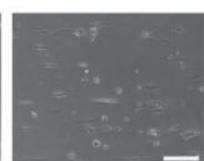

Figure 3. The microfluidic tool set for regulating external stem cell stimulation. (A) Cell responses can be manipulated by controlling medium perfusion and shear force. (i) An integrated device that utilizes valves and microposts to regulate fluid flow (reprinted with permission from Wiley [58]). (ii) Variations in micropillar number and spacing results in differences in velocity and shear stress profiles in the cultured region. (B) Hydrodynamic flow properties can result in compartmental delivery to cells growing within microchannels. (i) Half of an adherent ESC colony was labeled with Syto dye (reprinted with permission from RSC Publishing [65]). (ii) A device for compartmental delivery of differentiation factors to EBs (reprinted with permission from RSC Publishing [66]). (iii) This device was used to treat half an EB with retinoic acid resulting in differentiation to neurons on the treated half. (C) Biochemical gradients can guide differentiation in microfluidic devices. (i) A "Christmas Tree"-type gradient device used for stimulating multipotent neural stem cells (reprinted with permission from RSC Publishing [68]). (ii) This device was used to apply growth factors that maintain neural stem cells. Cells receiving low growth factor concentrations differentiate to astrocytes. (D) Topographic cues can provide instruction for cell adhesion/spreading, alignment, growth, and migration. (i) A nanotopographical pattern used to studying cellular alignment, cytoskeletal organization, focal adhesions, and mechanical properties of mesenchymal stem cells (reprinted with permission from Elsevier [75]). (ii) hESCs cultured on PDMS substrates with linear nanotopographic features, with staining for $\alpha$-SMA, a cytoskeleton protein. (iii) hESCs cultured on flat and linear nanotopograhy PDMS substrates, demonstrating cellular alignment/organization due to nanotopography (reprinted with permission from Elsevier [73]).

ent ESCs could be maintained in a pluripotent state in microfluidic channels for as long as conventional Petri dish cultures. Cells could then be differentiated, as shown by a disappearance of OCT3/4 expression. Additionally, this study demonstrated that it was possible to expose parts of the colonies to small molecules such as Syto dyes as well as the cell dissociation enzyme trypsin, opening up the possibility for multiple rounds of differentiation, cell harvesting, and regrowth into the harvested area. Fung et al. [66] also demonstrated that laminar flow could be used to apply selective biochemical treatment to a pluripotent stem cell EB (Fig. 3B). Half the EB was treated with retinoic acid, while the other half was not. Several days after the treatment, the retinoic acid-treated half expressed the neurofilament, a neuronal marker.

Other microfluidic systems can generate gradual gradients over cell cultures, potentially simulating the spatial and temporal presentation of molecules that stem cells are exposed to in vivo. Devices such as the "Christmas tree" gradient generator [67] have been developed for generating flow or flow-free gradients [64] to study signal propa- gation, cell-fate acquisition, and subsequent cellular dynamics within multipotent stem cell colonies [68] (Fig. 3C). Gradient-generating microfluidic systems can also provide temporal stimulation. These systems have been used to perturb cells to study metabolically regulated gene expression profiles [69]. In conventional culture systems, specific growth factors and conditions can induce differentiation of ESC-derived somatic cells; however, it is difficult to predict which cells will differentiate and of those differentiated cells it is difficult to control their lineage specification [70]. Integrated systems providing spatiotemporal stimulus control may give insights as to why heterogeneous differentiation occurs in cultures of ESCs. By providing methods to temporally and spatially treat cultures, a more homogeneous and reproducible population of differentiated cells may be obtained.

\subsection{Instructing with geometry and mechanics}

A variety of topographical substrate features and geometries can be used to provide structural information to di- 
rect cell attachment and behavior. Elasticity and other substrate properties are also important to consider for stem cell-based therapies since these factors can drive differentiation of stem cells that is independent of some biochemical differentiation cues [71]. Control of substrate rigidity in microfluidic systems was accomplished by Zaari et al. [72], by generating a continuous stiffness gradient. This was used to study migration and cell accumulation in regions with higher substrate stiffness within a microfluidic system.

Stem cells also have the capacity to sense micro-/ nanoscale features. For example, nanotopographical patterns in fibronectin-coated poly(di-methyl siloxane) (PDMS) induced hESC alignment and elongation [73] (Fig. 3D). Additionally, micro- or nanotopography can impact adhesion of cells through feature size and frequency of available features [74, 75]. These nanostructured microfluidic systems have recently been used to assess the role of both shear stress and nanotopography in cell attachment, spreading and migration, and may have a potential contribution in assessing cellular differentiation in future studies [76]. Protein topology can also provide spatial orientation cues. Different ECM structures, compositions, and their topographical coverage area change the mechanical properties presented to cells and consequently produce differential cellular responses [77-79]. It was established very early on that microfluidic systems could be used to pattern proteins in localized regions [56], and indeed they have been used extensively in a variety of cell-substrate interaction studies including assays for neurite extension [80] and selective cell adhesion to protein-deposited regions [81]. In addition to patterned topology, transitioning between the topographical influence between 2D and 3D systems has demonstrated dramatic changes in cellular responses and functions [82]. 3D gels within a microfluidic perfusion device offer the benefit that paracrine and autocrine factors produced by the cultured cells do not be immediately wash away, allowing for the intrinsic signaling from the cells of interest to be present for more physiologically realistic periods of time. Additionally, due to this diffusion barrier, researchers have taken advantage of generating stable biochemical gradients over EBs cultured in hydrogels [83].

\subsection{Recapitulating the niche- multimodal stimulation using microfluidics}

The ability to integrate multiple stimuli within one device would be an ideal technological advance that could produce optimal conditions for working with and manipulating pluripotent stem cells. We foresee an evolution of microfluidic devices incorporating multimodal stimulation, such as combined mechanical loading, biochemical signaling, and topographical patterning. This will provide cells with multiple cues, such that researchers can identify synergistic actions of the various stimulation paradigms.

\section{Microanalytical tools for stem cell biology}

A prominent goal of microfluidic systems is to transform lab-based testing systems into lab-on a-chip platforms for microanalytical approaches. Since integrated microfluidic devices are able to perform reproducible measurements more quickly and more sensitively, with smaller volumes than current conventional lab procedures, this opens the possibility to do single-cell high-throughput analyses, such as single-cell real-time quantitative PCR (qPCR) [84]. Other analytical tools that have been translated to the microscale include systems for genetic manipulation [85], cell fusion [86], and real-time analysis of stimulation-induced protein secretion [87]. Recently, similar technologies have been translated to use with pluripotent stem cells.

A high-throughput real-time $\mathrm{qPCR}$ microfluidic device was applied to determine co-regulation of a differentiation marker, miR-145, and pluripotent marker OCT4 during ESC differentiation [84]. With the ability to assess single cells as opposed to an averaged group sample, differences in cellular expression demonstrated that conventional methods masked gene co-regulation due to sample heterogeneity (Fig. 4A). Such examples suggest benefits of single-cell analysis to increase our understanding of population dynamics in pluripotent stem cell cultures and in tissues [88]. In addition to analyzing genetic expression, systems for gene transfer have been developed using microfluidic electroporation systems. Wang et al. [89] presented a serpentine mixer lined with electrodes that improved DNA uptake efficiency. This device was used to deliver genes to $\mathrm{MESC}$, demonstrating an approximately twofold increase in cell viability, with treated cells retaining morphology and adherent properties typical of adherent mESCs, whereas commercial systems resulted in a large proportion of cells having rounded morphology and loss of adherent properties.

Other systems developed for microfluidic cell testing have involved complex microfluidic chips composed of networks of channels regulated by integrated valves and pumps. One such device was developed to separate single cells from a bulk cell suspension and to deliver nanoliter volumes of reagents to the separated cells [90].

\section{Next steps}

\subsection{Induced pluripotent stem cells}

Induced pluripotent stem (iPS) cells were first generated in 2006 by transforming mouse fibroblasts with four factors- Oct3/4, Sox2, c-Myc, and Klf4 [91]. Within a year, follow-up studies reported that these cells could also be produced from adult human fibroblasts [92, 93]. These discoveries represent a major paradigm shift in the field of stem cell research, opening up possibilities for per- 


\section{A. Single Cell Gene Expression Profiling}
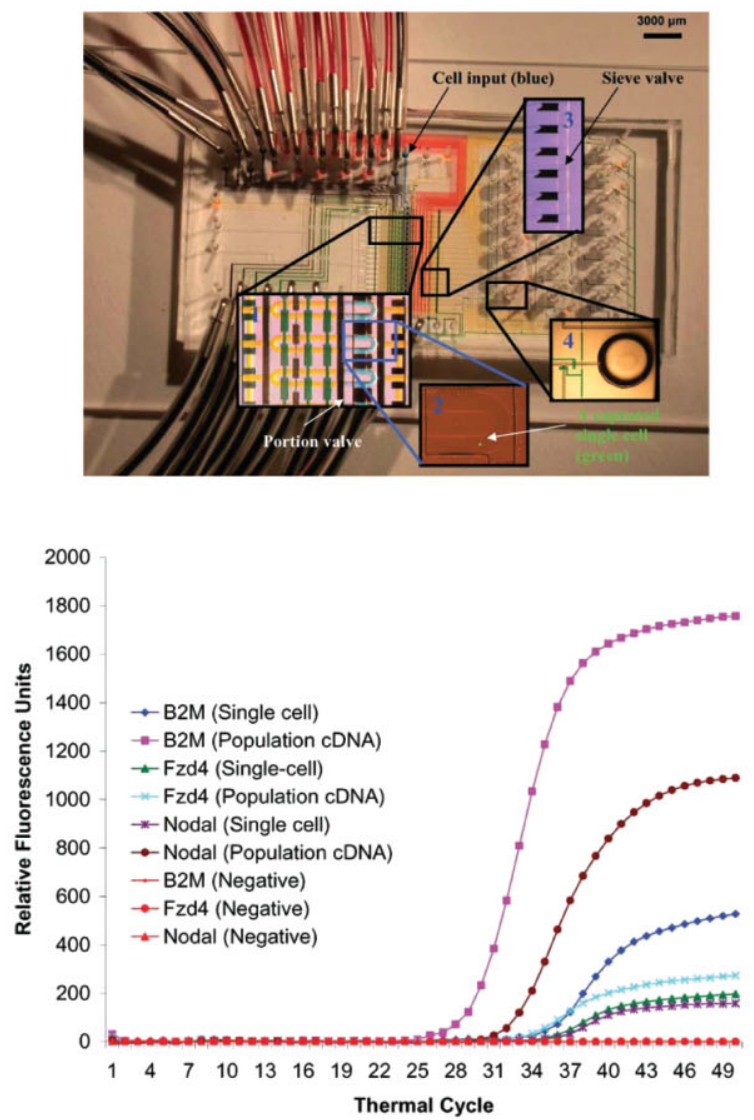

\section{B. Cell-Cell Fusion for Reprogramming}
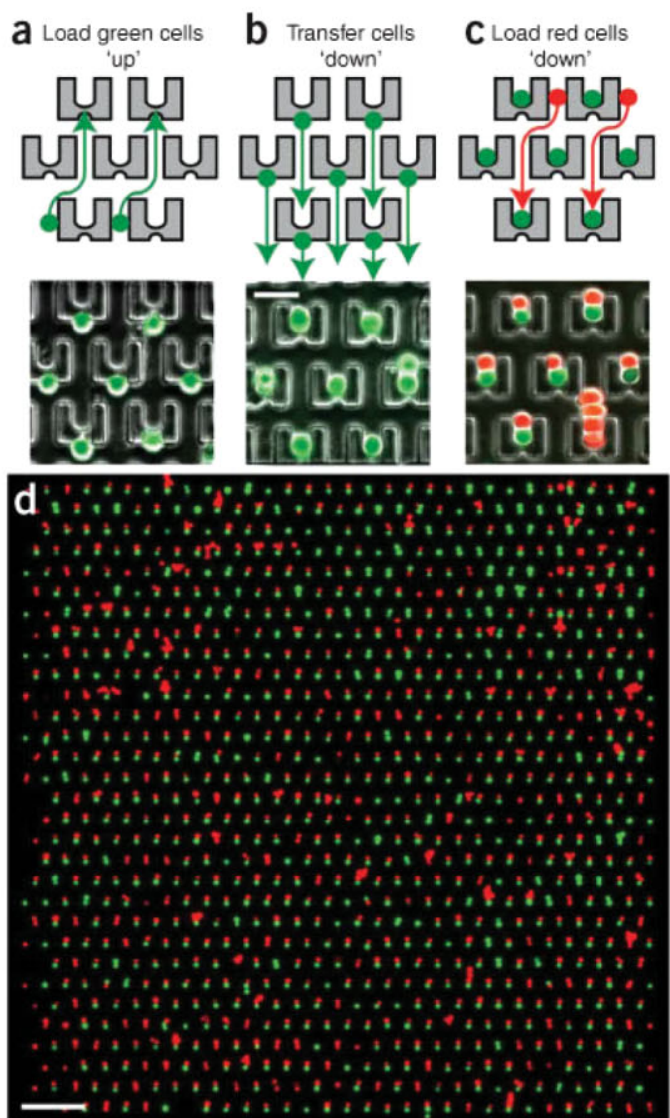

Figure 4. The microfluidic tool set for single-cell analysis and manipulation. (A) Microfluidic devices can be used to extract single-cell gene expression profiles from ESCs. The relative expression of stem cell markers differed dramatically between some single cells and a grouped cell population, indicating a large amount of heterogeneity within stem cell colonies (reprinted with permission from RSC Publishing [88]). (B) A microwell trapping device allowed 1:1 cell pairing of ESCs and somatic cells. This device improved cell fusion-based reprogramming of somatic cells by ESCs (reprinted with permission from Nature Publishing Group [86]).

sonalized stem cell medicine and diagnostics (using the patient's own cells), enabling development of disease models from human cells, and freeing the field from some ethical issues [94]. Considering the fact that after the cells have been induced to pluripotency the functional properties of iPS cells can be indistinguishable from ESCs, it is no surprise that many of the microtechnologies that are used with ESCs can also be applied to iPS cells. However, there are a number of recent reports that offer platforms specific to iPS cells that warrant discussion.

There are several limitations specific to iPS cells that can be improved upon using microtechnologies [95]. One challenge is that the cells derived from the reprogramming process are not a homogenous population. Most cells, in fact, do not get reprogrammed and may either resist transformation or exist in a partially reprogrammed state due to epigenetic modifications. Microfluidic technologies can aid in the reprogramming step in one of two ways, either by enhancing the efficiency at which cells are reprogrammed, or by characterizing subpopulations of cells with the end goal of purifying those cells that have been fully reprogrammed [86] (Fig. 4B)

A separate challenge involves developing platforms that allow researchers or clinicians to use these cells effectively. The technologies for iPS cells are mostly interchangeable with those used for differentiating other pluripotent stem cells. However, iPS cells offer additional possibilities for use as patient-specific disease models, and thus there is an emerging need to culture these cells in configurations that reproduce disease phenotypes in a format compatible with high-throughput therapeutic testing. A technical difficulty is that the differentiated cells must have the opportunity to interact with other cells or environmental factors in order to physiologically model certain diseases, but these factors are often not desired during stages of the differentiation process. Microfluidic and micropatterning tools capable of precisely position- 


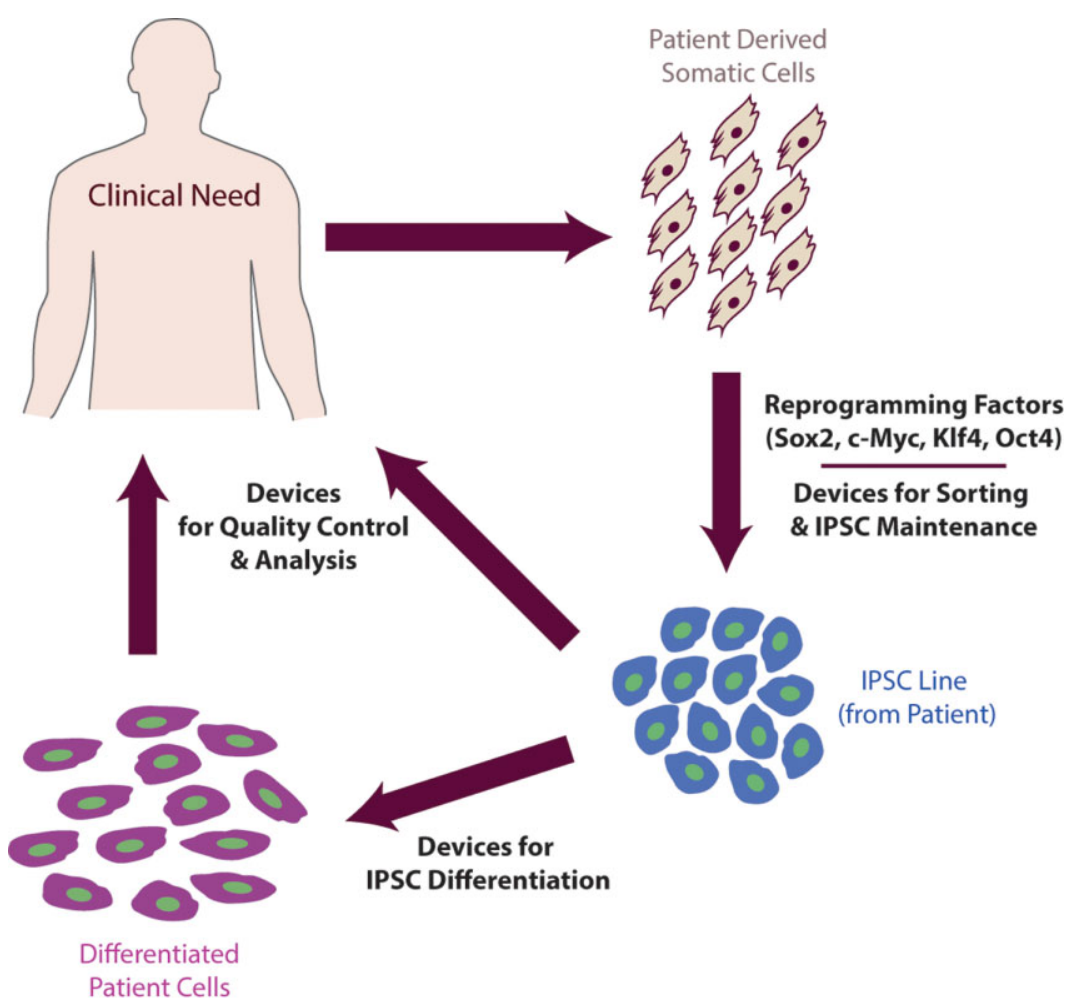

Figure 5. Future direction: microfluidics used on patient-derived iPS cells. Microfluidic systems may be implemented in personalized medicine by deriving patient somatic cells, and reprogramming them. Cells undergoing reprogramming treatment can then be processed by microfluidic devices to sort desired cells and maintain the iPS cell lines from the patient. iPS cells can be used directly for clinical needs or can be further differentiated through the use of microfluidic systems. Prior to clinical use, microfluidic devices provide an analytical platform for quality control of these cells. ing populations of cells or stimulating only the cells of interest following differentiation may be helpful for alleviating these constraints (Fig. 5).

\subsection{Scaling-up to meet commercial demands}

Complying with regulatory standards, such as good manufacturing procedures (GMP), is critical for stem cells to transition from a theoretical medical improvement to a therapeutic product. Adhering to GMP, cells should be maintained in closed systems whenever possible, and the use of biologically active exogenous substances should be minimized. Ideally, proteolytic enzymes for cellular disassociation and passaging will not be used for cell expansion, so as to remain GMP compliant, although this will likely become less of a limitation as recombinant enzymes free of animal products become increasingly available and gain approval. Mechanical disassociation provides a GMP compliant method of controlled passaging, but must be scaled up to meet the cellular production rate required for cell-based therapies. Wallman et al. [96] developed the Biogrid to scale up mechanical cutting to meet the cellular production rate; this microfluidic device drives cellular aggregates against microknives at the edges of a microgrid, fractioning large aggregates to smaller defined sizes, but requires that cells be growing in suspension. The Biogrid device was shown to have a more defined culture expansion rate and total cell quantity as compared to recombinant enzymatic passaging protocols.
Culture expansion also requires treating cells under the appropriate conditions. As pluripotent stem cells respond to shear stress from fluid flow, initial investigation of perfusion microbioreactors demonstrated that a window of appropriate fluid regimes exists; below certain flow rates, proliferation stops and cellular detachment occurs [97, 98], and above threshold flow rate proliferation improves, but is accompanied by cellular detachment and differentiation [98]. Using microfluidic systems to understand optimal culturing conditions, we come closer to meeting manufacturing compliance. The impact of scalable controlled microfluidic systems would be tremendous as suspension systems have recently been shown capable of deriving, expanding, and directing differentiation of iPS cells [99]. By integrating microfluidic components, the development of parallel microbioreactors capable of large scale growth, passaging, and monitoring of cells is possible for clinical scale production for pluripotent cellular therapeutics.

\section{Concluding remarks}

Microfluidic systems provide researchers with a tool set to spatially and temporally control the cellular microenvironment, and can be used to recapitulate in vivo stimuli and interrogate cell-cell, cell-ECM, and physicochemical interactions. Additionally, the integration of different elements within these devices provides the ability to 
manipulate cells, and to analyze bulk and individual cellular responses or molecular profiles to meet clinical requirements. Even though these systems have not been used extensively on research specific to pluripotent stem cells, translating these tool sets to pluripotent cells has begun and the benefits that microfluidic devices will transfer to developmental research will revolutionize our understanding of these cells. Through this understanding, we will enhance our ability to efficiently direct these cells into clinical applications.

The authors would like to thank the WCU (World Class University) program (No. R322008000200540) through the National Research Foundation of Korea (NRF) as funded by the Ministry of Education, Science and Technology (MEST) as well as the National Science Foundation Graduate Research Fellowship Program (to SCLP) under Grant No. DGE 1256260 (ID: 2011101670) for financial support.

Conflict of interest: Shuichi Takayama has stock options in 3D Biomatrix, a company that has licensed the technology to commercialize the high-throughput hangingdrop plates described in this review. The other authors declare no conflict of interest.

\section{References}

[1] Martin, G. R., Isolation of a pluripotent cell line from early mouse embryos cultured in medium conditioned by teratocarcinoma stem cells. Proc. Natl. Acad. Sci. USA 1981, 78, 7634-7638.

[2] Thomson, J. A., Itskovitz-Eldor, J., Shapiro, S. S., Waknitz, M. A. et al. Embryonic stem cell lines derived from human blastocysts. Science 1998, 282, 1145-1147.

[3] Hwang, N. S., Varghese, S., Elisseeff, J., Controlled differentiation of stem cells. Adv. Drug Deliv. Rev. 2008, 60, 199-214.

[4] Evans, M. J., Kaufman, M. H., Establishment in culture of pluripotential cells from mouse embryos. Nature 1981, 292, 154-156.

[5] Solter, D., From teratocarcinomas to embryonic stem cells and beyond: A history of embryonic stem cell research. Nat. Rev. Genet. 2006, 7, 319-327.

[6] Xu, R. H., Peck, R. M., Li, D. S., Feng, X. et al., Basic FGF and suppression of BMP signaling sustain undifferentiated proliferation of human ES cells. Nat. Methods 2005, 2, 185-190.

[7] Schuldiner, M., Eiges, R., Eden, A., Yanuka, O. et al., Induced neuronal differentiation of human embryonic stem cells. Brain Res. 2001, 913, 201-205.

[8] Nourse, M. B., Halpin, D. E., Scatena, M., Mortisen, D. J. et al., VEGF induces differentiation of functional endothelium from human embryonic stem cells: Implications for tissue engineering. Arterioscler. Thromb. Vasc. Biol. 2010, 30, 80-89.

[9] Toh, Y. C., Blagovic, K., Voldman, J., Advancing stem cell research with microtechnologies: Opportunities and challenges. Integr. Biol. (Camb.) 2010, 2, 305-325.

[10] Voldman, J., Gray, M. L., Schmidt, M. A., Microfabrication in biology and medicine. Annu. Rev. Biomed. Eng. 1999, 1, 401-425.

[11] El-Ali, J., Sorger, P. K., Jensen, K. F., Cells on chips. Nature 2006, 442, 403-411

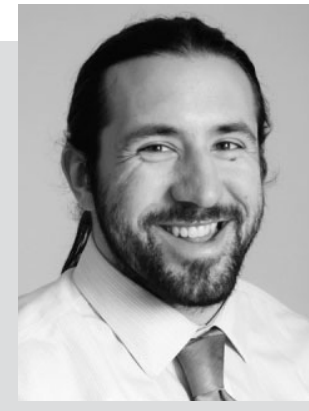

Sasha Cai Lesher-Perez is a doctoral student in Professor Shuichi Takayama's Micro/Nano/Molecular Biotechnology lab at the University of Michigan. Sasha Cai is an NSF Graduate Research Fellow, a Cellular Biotechnology Training Fellow, and a GEM Fellow. He started his graduate studies in 2009 after receiving his B.S. in Biomedical

Engineering from the University of Wisconsin, Madison. Sasha Cai's research focuses on utilizing microscale technologies to develop 3D early stage embryo models, with the goal of building physiological developmental models that generate accurate readouts and provide meaningful insights into preimplantation embryos to improve in vitro fertilization technology. Sasha Cai hopes to pursue a career in reproductive technology after he graduates.

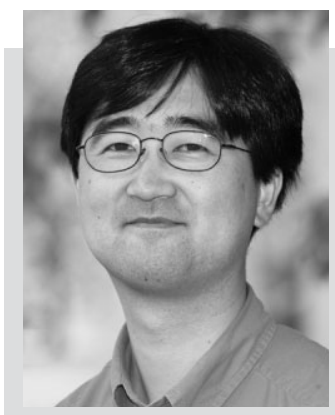

Shuichi Takayama is a Professor in the Department of Biomedical Engineering and the Macromolecular Science and Engineering Program at the University of Michigan. He is also WCU Visiting Professor at the Ulsan National Institute of Science and Technology (UNIST) in South Korea. His research interests (B.S. and M.S. from the University of Tokyo in 1994, his Ph.D. degree in chemistry from the Scripps Research Institute in 1998) started with organic synthesis of molecules that mediate biological chemical communication. Subsequently he became interested in evaluating the function of synthesized molecules in engineered bio-microsystems and pursued postdoctoral studies at Harvard University as a Leukemia and Lymphoma Society Fellow. His current research interests are micro/nanofluidics, cellular microenvironment engineering including stem cell cultures, and epigenetics. He constructs microfluidic models of the body such as artificial oviducts for enhanced in vitro fertilization treatment, microtissue engineered models of lung injury, and models of cancer metastasis for drug testing. He also develops aqueous two-phase system micropatterning technologies, self-switching fluidic circuits, and nanofluidic single-molecule DNA analysis systems. Honors include The Ralph E. Powe Junior Faculty Award and the NSF Career Award.

[12] Meyvantsson, I., Beebe, D. J., Cell culture models in microfluidic systems. Annu. Rev. Anal. Chem. (Palo Alto Calif.) 2008, 1, 423-449.

[13] Discher, D. E., Mooney, D. J., Zandstra, P. W., Growth factors, matrices, and forces combine and control stem cells. Science 2009, 324, 1673-1677.

[14] Wu, H. W., Lin, C. C., Lee, G. B., Stem cells in microfluidics. Biomicrofluidics 2011, 5, 13401

[15] van Noort, D., Ong, S. M., Zhang, C., Zhang, S. et al., Stem cells in microfluidics. Biotechnol. Prog. 2009, 25, 52-60. 
[16] Xu, C., Rosler, E., Jiang, J., Lebkowski, J. S. et al., Basic fibroblast growth factor supports undifferentiated human embryonic stem cell growth without conditioned medium. Stem Cells 2005, 23, 315-323.

[17] Ross, A. M., Nandivada, H., Ryan, A. L., Lahann, J., Synthetic substrates for long-term stem cell culture. Polymer 2012, 53, 2533-2539.

[18] Martin, G. R., Evans, M. J., Differentiation of clonal lines of teratocarcinoma cells: Formation of embryoid bodies in vitro. Proc. Natl. Acad. Sci. USA 1975, 72, 1441-1445.

[19] Kurosawa, H., Methods for inducing embryoid body formation: In vitro differentiation system of embryonic stem cells. J. Biosci. Bioeng. 2007, 103, 389-398.

[20] Lee, W. G., Ortmann, D., Hancock, M. J., Bae, H., Khademhosseini, A., A hollow sphere soft lithography approach for long-term hanging drop methods. Tissue Eng. Part C Methods 2010, 16, 249-259.

[21] Tung, Y. C., Hsiao, A. Y., Allen, S. G., Torisawa, Y. S. et al., Highthroughput 3D spheroid culture and drug testing using a 384 hanging drop array. Analyst 2011, 136, 473-478.

[22] Bratt-Leal, A. M., Carpenedo, R. L., McDevitt, T. C., Engineering the embryoid body microenvironment to direct embryonic stem cell differentiation. Biotechnol. Prog. 2009, 25, 43-51.

[23] Hwang, Y. S., Chung, B. G., Ortmann, D., Hattori, N. et al., Microwell-mediated control of embryoid body size regulates embryonic stem cell fate via differential expression of WNT5a and WNT11. Proc. Natl. Acad. Sci. USA 2009, 106, 16978-16983.

[24] Kim, C., Lee, K. S., Bang, J. H., Kim, Y. E. et al., 3-Dimensional cell culture for on-chip differentiation of stem cells in embryoid body. Lab Chip 2011, 11, 874-882.

[25] Torisawa, Y. S., Chueh, B. H., Huh, D., Ramamurthy, P. et al. , Efficient formation of uniform-sized embryoid bodies using a compartmentalized microchannel device. Lab Chip 2007, 7, 770-776.

[26] Jeong, G. S., Jun, Y., Song, J. H., Shin, S. H., Lee, S. H., Meniscus induced self organization of multiple deep concave wells in a microchannel for embryoid bodies generation. Lab Chip 2012, 12, 159-166.

[27] Karp, J. M., Yeh, J., Eng, G., Fukuda, J. et al., Controlling size, shape and homogeneity of embryoid bodies using poly(ethylene glycol) microwells. Lab Chip 2007, 7, 786-794.

[28] Khoury, M., Bransky, A., Korin, N., Konak, L. C. et al., A microfluidic traps system supporting prolonged culture of human embryonic stem cells aggregates. Biomed. Microdevices 2010, 12, 1001-1008.

[29] Kang, E., Choi, Y. Y., Jun, Y., Chung, B. G., Lee, S. H., Development of a multi-layer microfluidic array chip to culture and replate uniform-sized embryoid bodies without manual cell retrieval. Lab Chip 2010, 10, 2651-2654.

[30] Torisawa, Y. S., Mosadegh, B., Luker, G. D., Morell, M. et al., Microfluidic hydrodynamic cellular patterning for systematic formation of co-culture spheroids. Integr. Biol. (Camb.) 2009, 1, 649-654.

[31] Mironov, V., Reis, N., Derby, B., Review: Bioprinting: A beginning Tissue Eng. 2006, 12, 631-634.

[32] Xu, F., Sridharan, B., Wang, S., Gurkan, U. A. et al., Embryonic stem cell bioprinting for uniform and controlled size embryoid body formation. Biomicrofluidics 2011, 5, 22207.

[33] Anderson, D. G., Levenberg, S., Langer, R., Nanoliter-scale synthesis of arrayed biomaterials and application to human embryonic stem cells. Nat. Biotechnol. 2004, 22, 863-866.

[34] Tavana, H., Mosadegh, B., Takayama, S., Polymeric aqueous biphasic systems for non-contact cell printing on cells: Engineering heterocellular embryonic stem cell niches. Adv. Mater. 2010, 22, 26282631

[35] Tavana, H., Mosadegh, B., Zamankhan, P., Grotberg, J. B., Takayama, S., Microprinted feeder cells guide embryonic stem cell fate. Biotechnol. Bioeng. 2011, 108, 2509-2516.

[36] Westrom, L., Mardh, P. A., Mecklenburg, C. V., Hakansson, C. H., Studies on ciliated epithelia of the human genital tract. II. The mu- cociliary wave pattern of fallopian tube epithelium. Fertil. Steril. 1977, 28, 955-961.

[37] Lucitti, J. L., Jones, E. A., Huang, C., Chen, J. et al., Vascular remodeling of the mouse yolk sac requires hemodynamic force. Development 2007, 134, 3317-3326.

[38] Raya, A., Izpisua Belmonte, J. C., Left-right asymmetry in the vertebrate embryo: From early information to higher-level integration. Nat. Rev. Genet. 2006, 7, 283-293.

[39] Drummond, I. A., Cilia functions in development. Curr. Opin. Cell Biol. 2012, 24, 24-30.

[40] Tanaka, Y., Okada, Y., Hirokawa, N., FGF-induced vesicular release of Sonic hedgehog and retinoic acid in leftward nodal flow is critical for left-right determination. Nature 2005, 435, 172-177.

[41] Greco, T. L., Takada, S., Newhouse, M. M., McMahon, J. A. et al. Analysis of the vestigial tail mutation demonstrates that Wnt-3a gene dosage regulates mouse axial development. Genes Dev. 1996, 10, 313-324.

[42] Chen, Y., Huang, L., Russo, A. F., Solursh, M., Retinoic acid is enriched in Hensen's node and is developmentally regulated in the early chicken embryo. Proc. Natl. Acad. Sci. USA 1992, 89, 1005610059.

[43] Barkai, N., Shilo, B. Z., Robust generation and decoding of morphogen gradients. Cold Spring Harb. Perspect. Biol. 2009, 1, a001990.

[44] Goldin, S. N., Papaioannou, V. E., Paracrine action of FGF4 during periimplantation development maintains trophectoderm and primitive endoderm. Genesis 2003, 36, 40-47.

[45] Di-Gregorio, A., Sancho, M., Stuckey, D. W., Crompton, L. A. et al. BMP signalling inhibits premature neural differentiation in the mouse embryo. Development 2007, 134, 3359-3369.

[46] Kuijk, E. W., van Tol, L. T. A., Van de Velde, H., Wubbolts, R. et al. The roles of FGF and MAP kinase signaling in the segregation of the epiblast and hypoblast cell lineages in bovine and human embryos. Development 2012, 139, 871-882.

[47] Dorey, K., Amaya, E., FGF signalling: Diverse roles during early vertebrate embryogenesis. Development 2010, 137, 3731-3742.

[48] Baker, J., Liu, J. P., Robertson, E. J., Efstratiadis, A., Role of insulinlike growth factors in embryonic and postnatal growth. Cell 1993, 75, 73-82.

[49] Morriss-Kay, G. M., Ward, S. J., Retinoids and mammalian development. Int. Rev. Cytol. 1999, 188, 73-131.

[50] Miquerol, L., Langille, B. L., Nagy, A., Embryonic development is disrupted by modest increases in vascular endothelial growth factor gene expression. Development 2000, 127, 3941-3946.

[51] Mammoto, T., Ingber, D. E., Mechanical control of tissue and organ development. Development 2010, 137, 1407-1420.

[52] Vogel, V., Sheetz, M., Local force and geometry sensing regulate cell functions. Nat. Rev. Mol. Cell Biol. 2006, 7, 265-275.

[53] Wozniak, M. A., Chen, C. S., Mechanotransduction in development: A growing role for contractility. Nat. Rev. Mol. Cell Biol. 2009, 10, 34-43.

[54] Bendall, S. C., Stewart, M. H., Bhatia, M., Human embryonic stem cells: lessons from stem cell niches in vivo. Regen. Med. 2008, 3, 365-376.

[55] Kim, S. M., Lee, S. H., Suh, K. Y., Cell research with physically modified microfluidic channels: A review. Lab Chip 2008, 8, 1015-1023.

[56] Kane, R. S., Takayama, S., Ostuni, E., Ingber, D. E., Whitesides, G. M., Patterning proteins and cells using soft lithography. Biomaterials 1999, 20, 2363-2376.

[57] Beebe, D. J., Mensing, G. A., Walker, G. M., Physics and applications of microfluidics in biology. Annu. Rev. Biomed. Eng. 2002, 4, 261286

[58] Lee, J. M., Kim, J. E., Kang, E., Lee, S. H., Chung, B. G., An integrated microfluidic culture device to regulate endothelial cell differenti- 
ation from embryonic stem cells. Electrophoresis 2011, 32, 31333137.

[59] Adamo, L., Naveiras, O., Wenzel, P. L., McKinney-Freeman, S. et al., Biomechanical forces promote embryonic haematopoiesis. Nature 2009, 459, 1131-1135.

[60] Yamamoto, K., Sokabe, T., Watabe, T., Miyazono, K. et al., Fluid shear stress induces differentiation of Flk-1-positive embryonic stem cells into vascular endothelial cells in vitro. Am. J. Physiol. Heart Circ. Physiol. 2005, 288, H1915-1924.

[61] Toh, Y. C., Voldman, J., Fluid shear stress primes mouse embryonic stem cells for differentiation in a self-renewing environment via heparan sulfate proteoglycans transduction. FASEB J. 2011, 25, 1208-1217.

[62] Huang, H., Nakayama, Y., Oin, K., Yamamoto, K. et al., Differentiation from embryonic stem cells to vascular wall cells under in vitro pulsatile flow loading. J. Artif. Organs 2005, 8, 110-118.

[63] Abilez, O., Benharash, P., Mehrotra, M., Miyamoto, E. et al., A novel culture system shows that stem cells can be grown in 3D and under physiologic pulsatile conditions for tissue engineering of vascular grafts. J. Surg. Res. 2006, 132, 170-178.

[64] Keenan, T. M., Folch, A., Biomolecular gradients in cell culture systems. Lab Chip 2008, 8, 34-57.

[65] Villa-Diaz, L. G., Torisawa, Y. S., Uchida, T., Ding, J. et al, Microfluidic culture of single human embryonic stem cell colonies. Lab Chip 2009, 9, 1749-1755.

[66] Fung, W. T., Beyzavi, A., Abgrall, P., Nguyen, N. T., Li, H. Y., Microfluidic platform for controlling the differentiation of embryoid bodies. Lab Chip 2009, 9, 2591-2595.

[67] Jeon, N. L., Dertinger, S. K. W., Chiu, D. T., Choi, I. S. et al., Generation of solution and surface gradients using microfluidic systems. Langmuir 2000, 16, 8311-8316.

[68] Chung, B. G., Flanagan, L. A., Rhee, S. W., Schwartz, P. H. et al., Human neural stem cell growth and differentiation in a gradient-generating microfluidic device. Lab Chip 2005, 5, 401-406.

[69] Bennett, M. R., Pang, W. L., Ostroff, N. A., Baumgartner, B. L. et al., Metabolic gene regulation in a dynamically changing environment. Nature 2008, 454, 1119-1122.

[70] Boheler, K. R., Czyz, J., Tweedie, D., Yang, H. T. et al., Differentiation of pluripotent embryonic stem cells into cardiomyocytes. Circ. Res. 2002, 91, 189-201.

[71] Engler, A. J., Sen, S., Sweeney, H. L., Discher, D. E., Matrix elasticity directs stem cell lineage specification. Cell 2006, 126, 677-689.

[72] Zaari, N., Rajagopalan, P., Kim, S. K., Engler, A. J., Wong, J. Y., Photopolymerization in microfluidic gradient generators: Microscale control of substrate compliance to manipulate cell response. Adv. Mater. 2004, 16, 2133-2137.

[73] Gerecht, S., Bettinger, C. J., Zhang, Z., Borenstein, J. T. et al., The effect of actin disrupting agents on contact guidance of human embryonic stem cells. Biomaterials 2007, 28, 4068-4077.

[74] Arnold, M., Cavalcanti-Adam, E. A., Glass, R., Blummel, J. et al., Activation of integrin function by nanopatterned adhesive interfaces. ChemPhysChem 2004, 5, 383-388.

[75] Yim, E. K., Darling, E. M., Kulangara, K., Guilak, F., Leong, K. W., Nanotopography-induced changes in focal adhesions, cytoskeletal organization, and mechanical properties of human mesenchymal stem cells. Biomaterials 2010, 31, 1299-1306.

[76] Yang, Y., Kulangara, K., Sia, J., Wang, L., Leong, K. W., Engineering of a microfluidic cell culture platform embedded with nanoscale features. Lab Chip 2011, 11, 1638-1646.

[77] Flaim, C. J., Chien, S., Bhatia, S. N., An extracellular matrix microarray for probing cellular differentiation. Nat. Methods 2005, 2, 119125.

[78] Derda, R., Li, L., Orner, B. P., Lewis, R. L. et al., Defined substrates for human embryonic stem cell growth identified from surface arrays. ACS Chem. Biol. 2007, 2, 347-355.
[79] Chen, C. S., Mrksich, M., Huang, S., Whitesides, G. M., Ingber, D. E., Geometric control of cell life and death. Science 1997, 276, 14251428.

[80] Rhee, S. W., Taylor, A. M., Tu, C. H., Cribbs, D. H. et al., Patterned cell culture inside microfluidic devices. Lab Chip 2005, 5, 102-107.

[81] Khademhosseini, A., Suh, K. Y., Jon, S., Eng, G. et al., A soft lithographic approach to fabricate patterned microfluidic channels. Anal. Chem. 2004, 76, 3675-3681.

[82] Abbott, A., Cell culture: Biology's new dimension. Nature 2003, 424, 870-872.

[83] Barkefors, I., Thorslund, S., Nikolajeff, F., Kreuger, J., A fluidic device to study directional angiogenesis in complex tissue and organ culture models. Lab Chip 2009, 9, 529-535.

[84] White, A. K., VanInsberghe, M., Petriv, O. I., Hamidi, M. et al., Highthroughput microfluidic single-cell RT-qPCR. Proc. Natl. Acad. Sci. USA 2011, 108, 13999-14004.

[85] Fei, Z., Hu, X., Choi, H. W., Wang, S. et al., Micronozzle array enhanced sandwich electroporation of embryonic stem cells. Anal. Chem. 2010, 82, 353-358.

[86] Skelley, A. M., Kirak, O., Suh, H., Jaenisch, R., Voldman, J., Microfluidic control of cell pairing and fusion. Nat. Methods 2009, 6, 147-152.

[87] Shackman, J. G., Reid, K. R., Dugan, C. E., Kennedy, R. T., Dynamic monitoring of glucagon secretion from living cells on a microfluidic chip. Anal. Bioanal. Chem. 2012, 402, 2797-2803.

[88] Zhong, J. F., Chen, Y., Marcus, J. S., Scherer, A. et al., A microfluidic processor for gene expression profiling of single human embryonic stem cells. Lab Chip 2008, 8, 68-74.

[89] Wang, S., Zhang, X., Wang, W., Lee, L. J., Semicontinuous flow electroporation chip for high-throughput transfection on mammalian cells. Anal. Chem. 2009, 81, 4414-4421.

[90] Wheeler, A. R., Throndset, W. R., Whelan, R. J., Leach, A. M. et al., Microfluidic device for single-cell analysis. Anal. Chem. 2003, 75, 3581-3586

[91] Takahashi, K., Yamanaka, S., Induction of pluripotent stem cells from mouse embryonic and adult fibroblast cultures by defined factors. Cell 2006, 126, 663-676.

[92] Takahashi, K., Tanabe, K., Ohnuki, M., Narita, M. et al., Induction of pluripotent stem cells from adult human fibroblasts by defined factors. Cell 2007, 131, 861-872.

[93] Yu, J., Vodyanik, M. A., Smuga-Otto, K., Antosiewicz-Bourget, J. et al., Induced pluripotent stem cell lines derived from human somatic cells. Science 2007, 318, 1917-1920.

[94] Inoue, H., Yamanaka, S., The use of induced pluripotent stem cells in drug development. Clin. Pharmacol. Ther. 2011, 89, 655-661.

[95] Saha, K., Jaenisch, R., Technical challenges in using human induced pluripotent stem cells to model disease. Cell Stem Cell 2009, 5, 584-595.

[96] Wallman, L., Akesson, E., Ceric, D., Andersson, P. H. et al., Biogrid a microfluidic device for large-scale enzyme-free dissociation of stem cell aggregates. Lab Chip 2011, 11, 3241-3248.

[97] Korin, N., Bransky, A., Dinnar, U., Levenberg, S., Periodic „flow-stop“ perfusion microchannel bioreactors for mammalian and human embryonic stem cell long-term culture. Biomed. Microdevices 2009, 11, 87-94.

[98] Titmarsh, D., Hidalgo, A., Turner, J., Wolvetang, E., Cooper-White, J., Optimization of flowrate for expansion of human embryonic stem cells in perfusion microbioreactors. Biotechnol. Bioeng. 2011, 108, 2894-2904.

[99] Fluri, D. A., Tonge, P. D., Song, H., Baptista, R. P. et al., Derivation, expansion and differentiation of induced pluripotent stem cells in continuous suspension cultures. Nat. Methods 2012, 9, 509-516. 